Bull. Mater. Sci., Vol. 35, No. 6, November 2012, pp. 989-995. (c) Indian Academy of Sciences.

\title{
Effect of T6 heat treatment on damping characteristics of Al/RHA composites
}

\author{
D SIVA PRASAD ${ }^{\dagger, *}$ and A RAMA KRISHNA \\ $\dagger$ Department of Mechanical Engineering, Gitam University, Visakhapatnam 530 045, India \\ ¥Department of Mechanical Engineering, Andhra University, Visakhapatnam 530 003, India
}

MS received 28 August 2011; revised 27 November 2011

\begin{abstract}
In the present work, effect of T6 heat treatment on the damping behaviour of aluminum/rice husk ash (RHA) composites fabricated by vortex method was studied using dynamic mechanical analyser (DMA) at frequencies ranging from $1 \mathrm{~Hz}$ to $25 \mathrm{~Hz}$ at room temperature under three-point bending test mode. The matrix material for the present work was A356.2 and reinforced with different weight $\%$ of 4, 6 and 8 rice husk ash particles. It was observed that composite exhibits high damping capacities than unreinforced alloy and increases with increase in weight $\%$ and the storage modulus increases with the addition of RHA particles but decreases with the increase in weight $\%$. The heat treated composites exhibit higher damping capacity than the composites without heat treatment and increases with the increase in weight $\%$ of the reinforcement and loss in the storage modulus was observed and further decreases with the increase in the weight $\%$ of reinforcement. The related mechanisms were also discussed.
\end{abstract}

Keywords. Rice husk ash; A356.2; DMA.

\section{Introduction}

Damping capacity is a manifestation of anelastic behaviour, i.e. a time-dependent response to an applied load. Structural materials that exhibit high damping capacities are desirable for mechanical vibration suppression and acoustic noise attenuation. Mechanical vibrations often affect proper functioning of equipment. For instance, fatigue failure is common in structures exposed to uncontrolled vibrations. There are also health concerns to humans from the excessive noise being transmitted from such sources. Damping capacity, a material's ability to absorb and dissipate mechanical vibrations, is usually low in structural metals. As a result, vibration attenuation is normally achieved by using external devices to absorb and transmit vibrations from structures. Metal matrix composite technology, however, offers the possibility of enhancing damping characteristics of structural metals by incorporating high damping materials as reinforcements. $\mathrm{SiC}, \mathrm{Al}_{2} \mathrm{O}_{3}$ and graphite are the most frequently used reinforcements in these MMCs.

Coulomb was the first to observe damping in 1784 under torsional vibration condition. Since then much investigation have been made and various damping mechanisms were discovered in metallic materials. Rohatgi et al (1994) investigated the damping capacity of graphite and silicon carbide particulate reinforced $\mathrm{Al}$ alloy composites. The damping capacity of graphite/Al alloy composites increased

\footnotetext{
*Author for correspondence (dorasivaprasad@gmail.com)
}

with the volume percentage of graphite within the range studied. However, no obvious improvements in damping capacity were observed by dispersion of silicon carbide in aluminum alloy. In contrast, Wang and Zhu (1998) showed that the presence of 13 vol. \% of SiC $p$ clearly increases the damping characteristics compared to the monolithic material condition and Srikanth and Gupta (2002) reported that the damping capacity of pure magnesium matrix was improved in the presence of $\mathrm{SiC}$ particulates, and increased with the increase of the proportion of $\mathrm{SiC}$ particulates. Wu et al (2006) investigated the damping properties of the hollow sphere fly ash/6061 Al composites. The damping capacity of the fly ash/6061 Al composite can reach $3.2 \times 10^{-2}$, which is in the high damping capacity level. The damping behaviour of $\mathrm{FeAl}_{3}$ reinforced composites and base alloy was studied by Jia (2007). The $\mathrm{Al} / \mathrm{FeAl}_{3}$ composites were found to exhibit higher damping capacity compared to Al matrix over the studied temperature range, and its damping capacity, as a function of temperature, increases with increasing temperature. The damping mechanisms associated with $\mathrm{Al} / \mathrm{FeAl}_{3} \quad \mathrm{MMCs}$ were ascribed to dislocation damping at low testing temperatures, and to dislocation damping and interface damping at high testing temperatures. Sudarshan and Surappa (2008) also showed that fly ash/A356 MMCs, with proper volume fraction of fly ash particulates, exhibited improved ambient temperature damping capacity, as well as the hardness, elastic modulus, and $0.2 \%$ proof stress, compared to unreinforced alloy.

According to Taya and Arsenault (1989), damping mechanism occurs due to high density of dislocations at the matrix-reinforcement interface. The energy dissipation arises 
due to increased relative atomic movement by the dislocations in a crystalline lattice, which interfere with homogenous deformation of the bulk material under applied stress. Thus the induced strain lags behind the stress causing increased damping characteristics. Based on the works of Bishop and Kinra (1995), addition of ceramic particulate improves the elasto thermodynamic damping capability of the metallic matrix. Their results also showed that the thermoelastic damping is only significant when materials are tested above a vibration frequency of $100 \mathrm{~Hz}$. Based on the works of Dunand and Mortensen (1991), the annular plastic zone is induced around the reinforcement and is due to thermal mismatch caused due to difference in coefficient of thermal expansion between the $\mathrm{Al}$ and reinforcement phases and proposed a simple model for the size of the plastic zone with an assumption of elastic perfectly plastic material behaviour. Carreno-Morelli et al (2000) proposed a model to determine the damping due to plastic zone. The increase in damping through the interfacial slip was studied by Nelson and Hancock (1978).

Although many researchers have studied the damping behaviour of aluminum alloys and conventional aluminum alloy-based composites, there is dearth of information on the damping characteristics of A356.2 alloy and the effect of rice husk ash particulates addition on the damping behaviour. The main objective of this work is to understand the damping behaviour of cast aluminum alloy, A356.2 and its composites reinforced with rice husk ash particulates. Damping measurements were done using dynamic mechanical analyser for the unreinforced alloy and the A356-2/RHA composites with and without heat treatment.

\section{Experimental}

\subsection{Pretreatment of $R H A$}

RHA particles obtained from local sources contained moisture, inorganic matter and some carbonaceous material. In order to free it from inorganic matter, some carbonaceous material pretreatment was done to RHA. In the present study, rice husk was procured from local sources and was thoroughly washed with water to remove the dust and dried at room temperature for 1 day. The rice husk was then heated to $200^{\circ} \mathrm{C}$ for $1 \mathrm{~h}$ to remove the moisture. It was then heated to $600{ }^{\circ} \mathrm{C}$ for $12 \mathrm{~h}$ to remove the carbonaceous material. After this operation, the colour changed from black to grayish white. The silica-rich ash, thus obtained, was used as a reinforcement material in the preparation of composites. The chemical composition of RHA after the above treatments is shown in table 1 .

\subsection{Preparation of composites}

The matrix for the preparation of composites was A356.2 with the chemical composition shown in table 2. Initially,
Table 1. Chemical composition of RHA.

\begin{tabular}{lc}
\hline Constituent & $\%$ \\
\hline Silica $-\mathrm{SiO}_{2}$ & $90 \cdot 23$ \\
Graphite & 4.77 \\
Calcium oxide $-\mathrm{CaO}$ & 1.58 \\
Magnesium oxide $-\mathrm{MgO}$ & 0.53 \\
Potassium oxide $-\mathrm{K}_{2} \mathrm{O}$ & $0 \cdot 39$ \\
Ferric oxide $-\mathrm{Fe}_{2} \mathrm{O}_{3}$ & $0 \cdot 21$ \\
\hline
\end{tabular}

Table 2. Chemical composition of A356.2 Al alloy matrix.

\begin{tabular}{lccccccr}
\hline $\mathrm{Si}$ & $\mathrm{Fe}$ & $\mathrm{Cu}$ & $\mathrm{Mn}$ & $\mathrm{Mg}$ & $\mathrm{Zn}$ & $\mathrm{Ni}$ & $\mathrm{Ti}$ \\
\hline $6.5-7.5$ & 0.15 & 0.03 & 0.10 & 0.4 & 0.07 & 0.05 & 0.1 \\
\hline
\end{tabular}

A356.2 Al with a theoretic density of $2760 \mathrm{~kg} / \mathrm{m}^{3}$ alloy was fed into the graphite crucible and heated to $750{ }^{\circ} \mathrm{C}$ till the entire alloy in the crucible was melted. The reinforcement particles (RHA) were preheated to $800^{\circ} \mathrm{C}$ for $1 \mathrm{~h}$ before incorporation into the melt. After the molten metal was fully melted, degassing tablet was added to reduce porosity. The stirrer made up of stainless steel was lowered into the melt slowly to stir the molten metal at a speed of $700 \mathrm{rpm}$. The preheated RHA particles were added into the molten metal at a constant rate during stirring time. The stirring was continued for another 5 min even after the completion of particle feeding. The mixture was poured into the mold which was also preheated to $500^{\circ} \mathrm{C}$ for 30 min to obtain uniform solidification. Using the process 4,6 and $8 \%$ by weight, RHA particle-reinforced composites were produced.

\subsection{T6 heat treatment}

The samples prepared for damping measurement were heated up to $535^{\circ} \mathrm{C} \pm 5^{\circ} \mathrm{C}$ for $6 \mathrm{~h}$ in a furnace followed by quenching in cold water and this condition is termed solutionized condition. The ageing temperature was maintained at $155^{\circ} \mathrm{C}$ for $6-8 \mathrm{~h}$.

\subsection{Microstructure analysis}

Microstructural characterization studies were conducted on RHA reinforced samples in order to investigate distribution of rice husk ash particles retained in the MMC. This was accomplished by using scanning electron microscope. In the present study, JSM-6610LV scanning electron microscope (SEM) equipped with energy dispersive $\mathrm{X}$-ray analyser (EDX) was used to study microstructure of the A356.2/RHA composites. The samples of unreinforced and A356.2/RHA composites for SEM was cut from tensile specimens and were ground by means of abrasive papers (P220, P400, P800, 


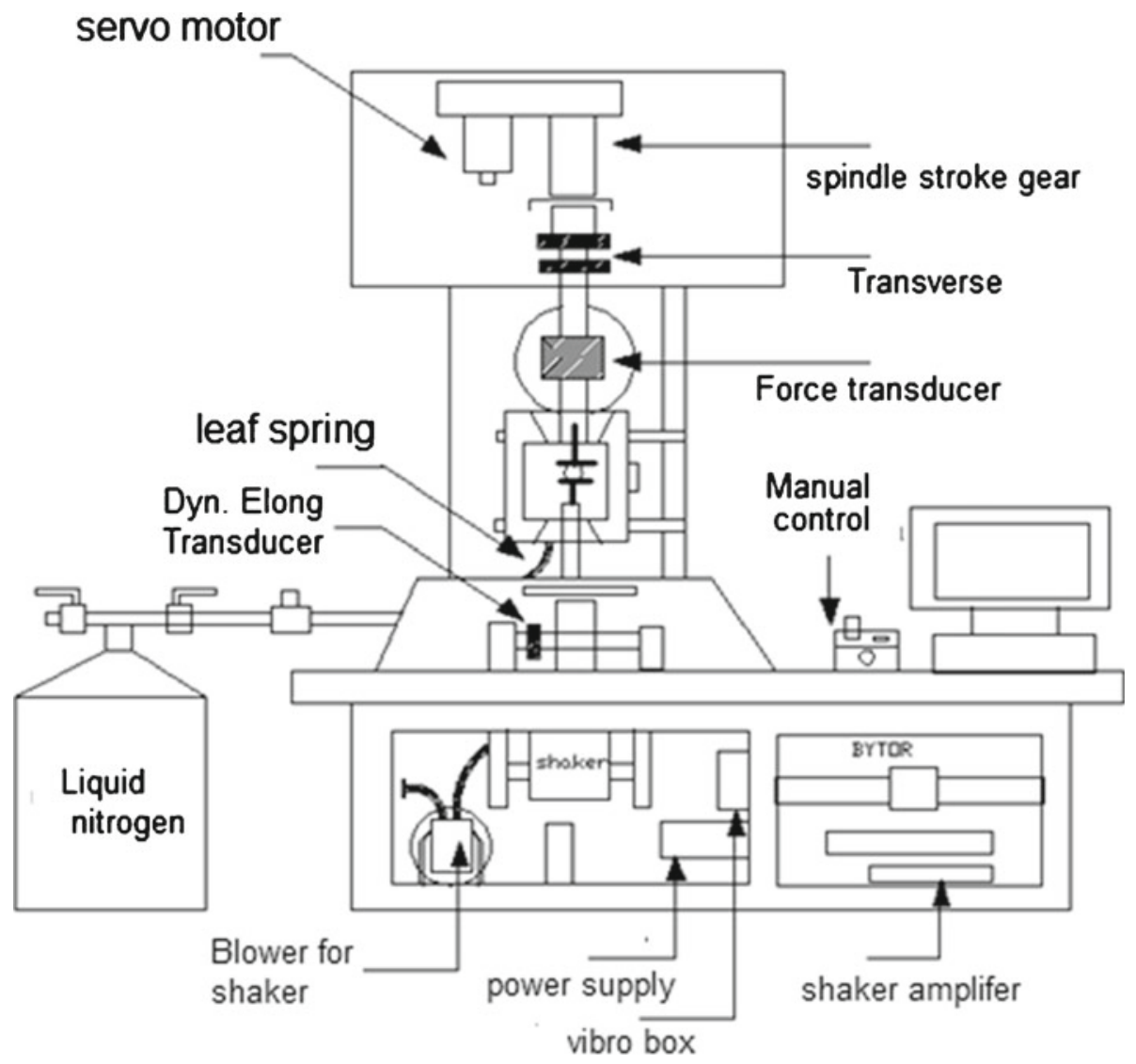

Figure 1. Schematic diagram of GABO Eplexor DMA.

P1200) followed by rotating disc cloth polishing. Keller's reagent ( $95 \mathrm{ml}$ water, $2.5 \mathrm{ml} \mathrm{HNO}_{3}, 1.5 \mathrm{ml} \mathrm{HCl}, 1.0 \mathrm{ml} \mathrm{HF}$ ), very popular general purpose reagent for $\mathrm{Al}$ and $\mathrm{Al}$ alloys, was used as an etching agent. The samples were immersed for 10-20 s in the above solution and then washed with warm water, followed by dipping in concentrated $\mathrm{HNO}_{3}$.

\subsection{Damping measurements}

The loss tangent and dynamic modulus were measured using a GABO Eplexor dynamic mechanical analyser for composites with and without heat treatment. DMA itself contained dynamic vibrator system with strain sensor, the static servo drive, the temperature chamber with thermocouples, the exchangeable force transducers as well as the power supply, amplifiers and the heart of the system, a 32-bit real-time multitask processing computer. A schematic view of the system is shown in figure 1 .

\subsection{Specimen preparation for damping measurements}

Rectangular bending beam specimens for damping measurements with dimensions of $30 \times 12 \times 1.5 \mathrm{~mm}$ were machined out using an Ultra cut $843 /$ Ultra cut $\mathrm{f} 2 \mathrm{CNC}$ wire electric discharge machine. The heat treatment of specimens was performed in an electric furnace.

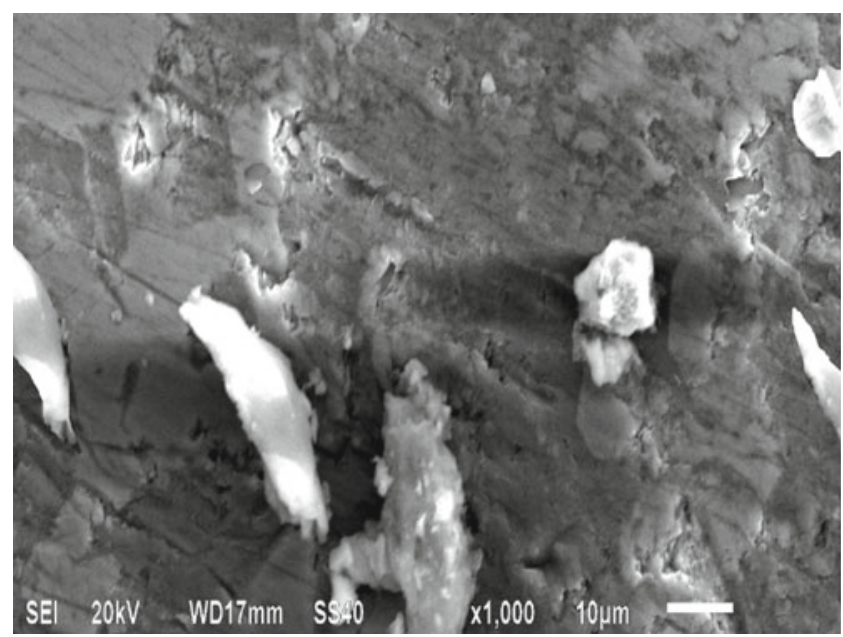

Figure 2. SEM picture of A356-2/RHA composites.

\section{Results}

\subsection{Microstructure analysis}

A representative view of microstructure of the A356.2 alloy based composite with $6 \%$ RHA is shown in figure 2. In the A356-2/RHA composite, distribution of RHA particles is rather uniform, and RHA particles disperse in the matrix 


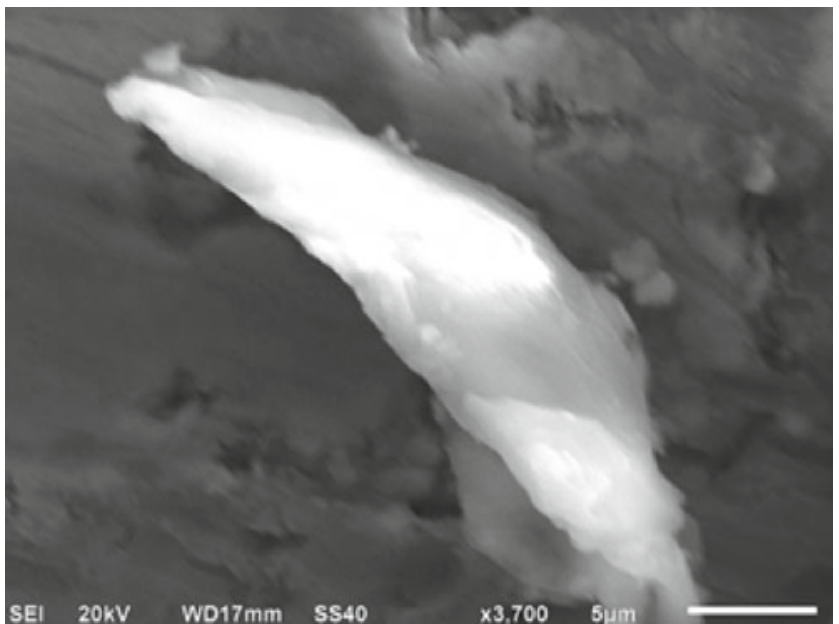

Figure 3. Scanning electron micrograph showing A356.2 matrix and RHA interface.

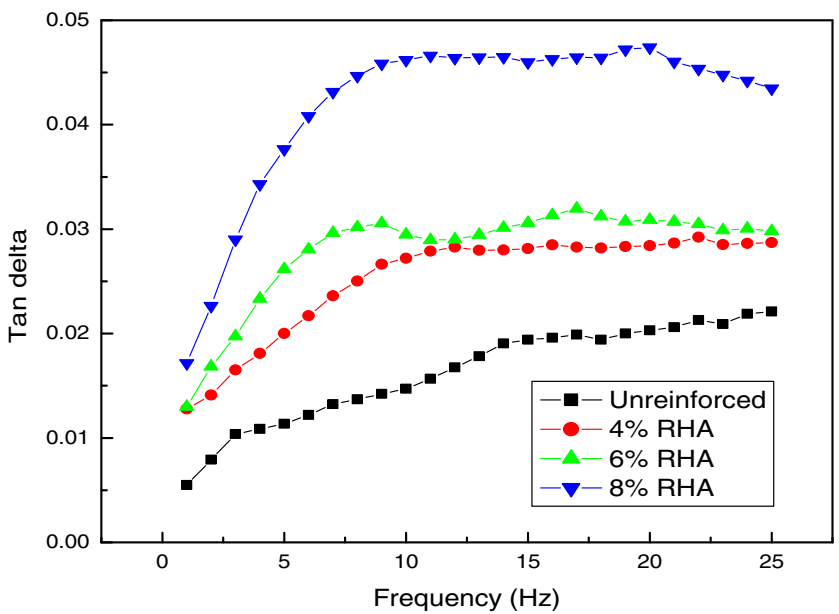

Figure 4. Comparison of $\tan \delta$ for unreinforced, $4 \%, 6 \%$ and $8 \%$ RHA composites.

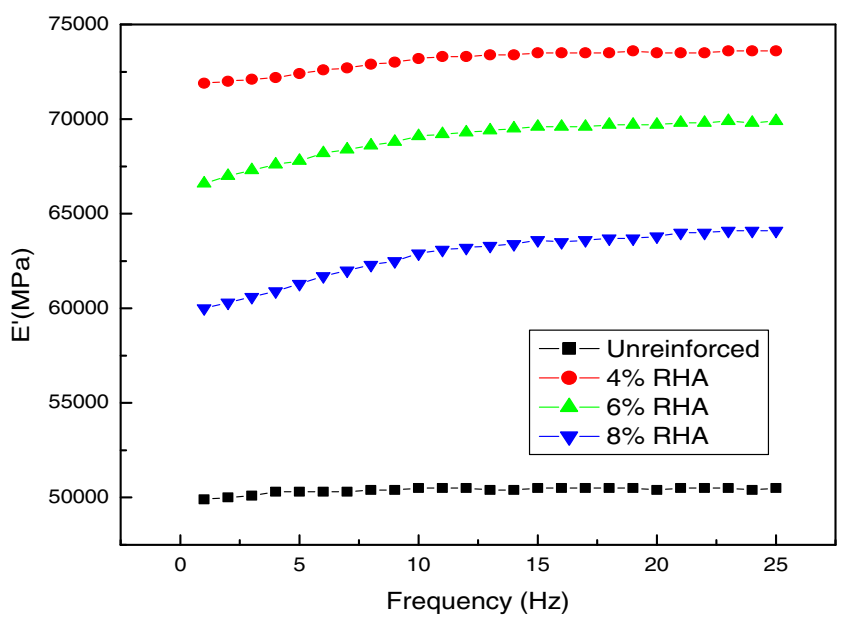

Figure 5. Comparison of $E^{1}$ for unreinforced, $4 \%, 6 \%$ and $8 \%$ RHA composites.

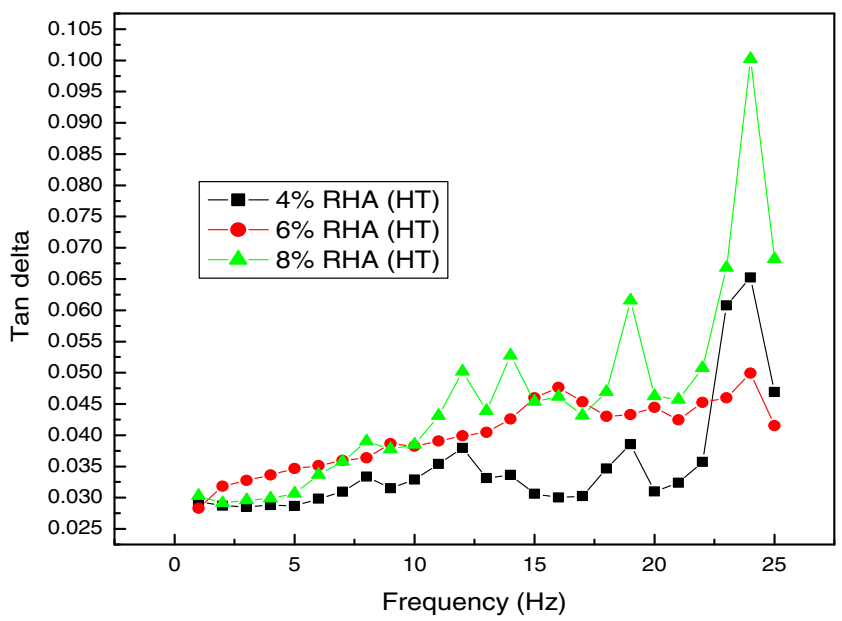

Figure 6. Comparison of $\tan \delta$ for $4 \%, 6 \%$ and $8 \%$ RHA heat treated (HT) composite.

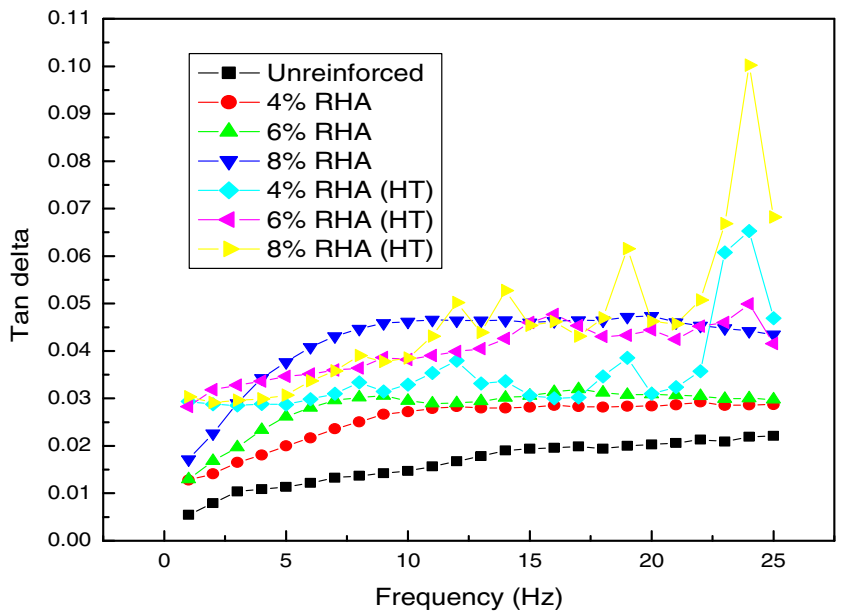

Figure 7. Comparison of $\tan \delta$ for $4 \%, 6 \%$ and $8 \%$ RHA with and without heat treated (HT) composite.

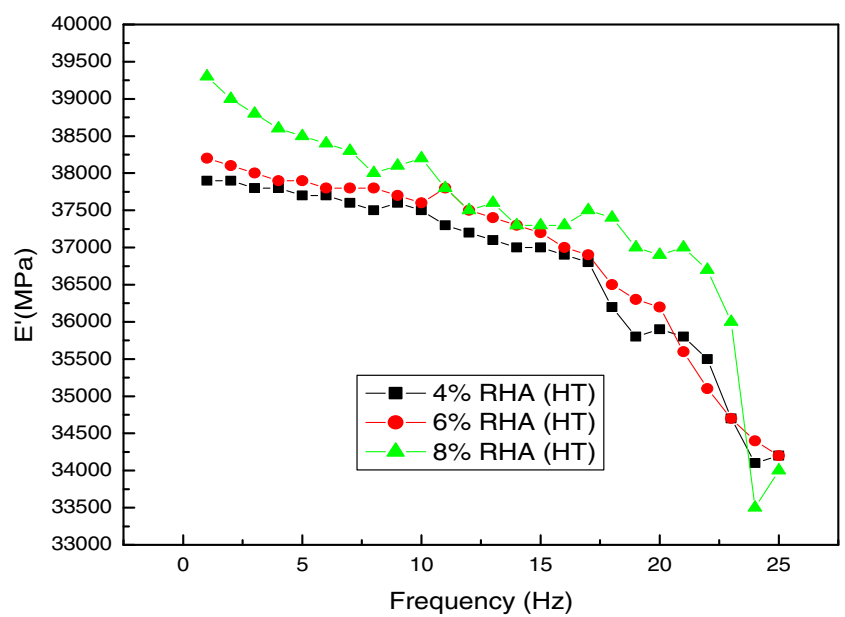

Figure 8. Comparison of $E^{1}$ for $4 \%, 6 \%$ and $8 \%$ RHA heat treated (HT) composite. 
separately; no evident agglomeration of RHA particles can be found. On the basis of the microstructure of the interface between the matrix and reinforcement (figure 3), RHA particles are bonded with aluminum matrix intimately, and no evidence of significant chemical reaction between A356-2/RHA is found.

\subsection{Damping measurements}

Figure 4 shows damping capacities of the unreinforced, $4 \%, 6 \%$ and $8 \%$ RHA composites measured using dynamic mechanical analyser in the frequency ranging from $1 \mathrm{~Hz}$ to $25 \mathrm{~Hz}$ under three-point bending method. As A356.2 is a low damping material $(\tan \delta=0.00549$ at $1 \mathrm{~Hz}$ ) which can be seen from the experimental data (figure 4) and needs to enhance the damping capacity for many industrial applications, an attempt is made to enhance its damping capacity by

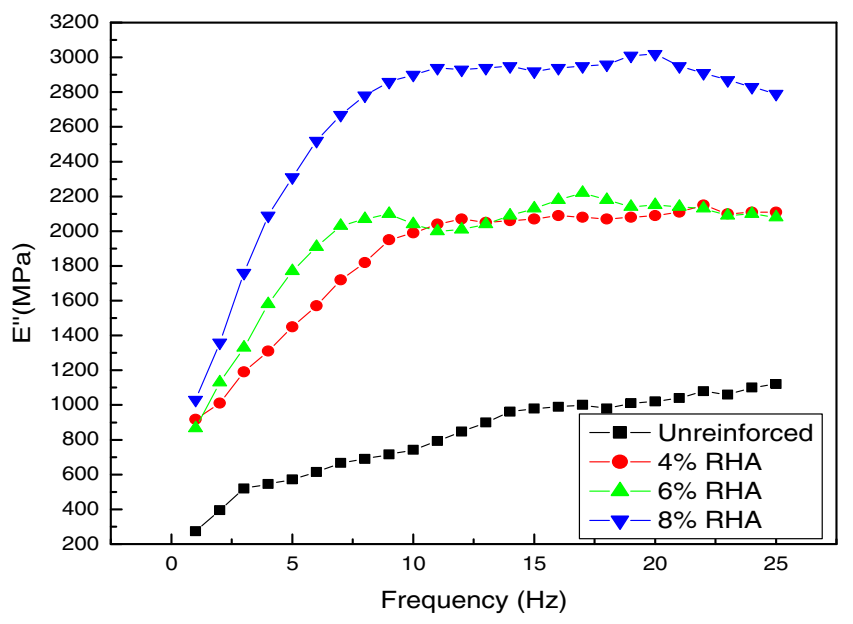

Figure 9. Comparison of $E^{11}$ for unreinforced, $4 \%, 6 \%$ and $8 \%$ RHA composites.

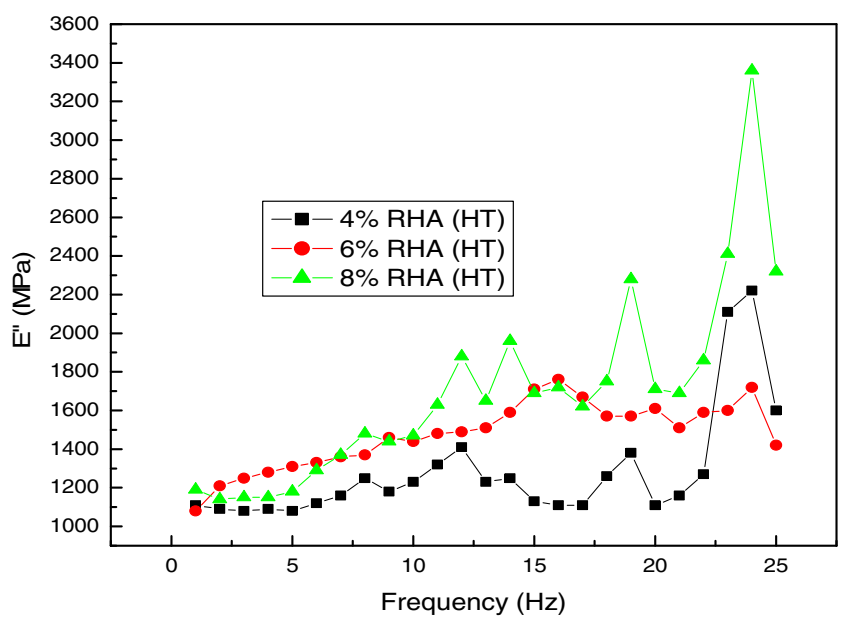

Figure 10. Comparison of $E^{11}$ for $4 \%, 6 \%$ and $8 \%$ RHA heat treated (HT) composite. incorporating RHA particles in the matrix. It can be clearly seen that the damping capacity increases with the increase in weight fraction of RHA particles. The storage modulus for untreated composites increases with the addition of reinforcement but decreases with increase in weight fraction (figure 5). The damping capacities of the heat treated composites increase and it should be noted that the damping capacity spectra of the composite exhibit peaks (figures 6 and 7). Similar peaks are not found in the untreated and unreinforced composites. The storage modulus of heat treated composites falls sharply and decreases with the weight $\%$ of reinforcement (figure 8). The variation of loss modulus with frequency was also shown in figures 9 and 10.

\section{Discussion}

The damping in crystalline materials can be attributed to several mechanisms such as thermoelastic damping, intrinsic damping, interfacial damping, point defect damping, grain boundary and thermal mismatch damping and others. As a reinforcing phase is added to the matrix, many of these factors may be augmented while additional new mechanisms may appear. The following discussion will address the factors which are thought to have been active in the A356.2/RHA MMCs studied.

\subsection{Thermoelastic damping}

The classical theory of thermoelastic damping says when a beam is flexed, one side of the beam is in tension and the other side is in compression. The side in compression gets slightly warmer and the side in tension gets slightly cooler due to the coupled nature of thermal and mechanical domains. A temperature gradient is formed across the beam giving rise to heat flow (for nonzero thermal conductivity) and this heat flow is an irrecoverable energy loss that limits the quality factor $\left(Q^{-1}\right)$ of the beam. The thermoelastic damping is related to the thermal relaxation constant $\Delta$, and relaxation time, $\tau$, by the following equation:

$$
Q^{-1}=\tan \delta=\frac{\Delta \omega \tau}{1+\omega^{2} \tau^{2}},
$$

where

$$
\Delta=\frac{E \alpha^{2} T}{C_{\mathrm{v}}},
$$

and

$$
\tau=\frac{C_{\mathrm{v}} h^{2}}{\pi^{2} k},
$$

where $E$ is the Young's modulus, $\alpha$ the coefficient of thermal expansion (CTE), $T$ the absolute temperature, $C_{\mathrm{v}}$ the specific heat per unit volume, $\omega$ the angular frequency $(\omega=$ $2 \pi f), h$ the beam thickness and $k$ the thermal conductivity. According to Taya and Arsenault (1989), if the volume fraction is low the quantities for a particulate reinforced MMC 
are comparable to their counterparts of the matrix alloy. Using the relevant data for A356.2 $\left(\alpha=21.4 \times 10^{-6} /{ }^{\circ} \mathrm{C}\right.$, $k=151 \mathrm{~J} / \mathrm{sm}^{\circ} \mathrm{C}, C_{\mathrm{v}}=963 \mathrm{~J} / \mathrm{kg}^{\circ} \mathrm{C} ; E=72.4 \mathrm{GPa}, h=$ $1.5 \mathrm{~mm}$ ) yields $\tan \delta$ in the order $9.1 \times 10^{-4}$ at a frequency of $1 \mathrm{~Hz}$ at room temperature which is not in good agreement with the experimental results. The extent of thermoelastic damping in particulate reinforced aluminum composites was studied by Bishop and Kinra (1995). Their results show thermoelastic damping is only significant when materials are tested above a vibration frequency of $100 \mathrm{~Hz}$. The present work involves frequency sweep in the range from $1 \mathrm{~Hz}$ to $25 \mathrm{~Hz}$ hence it is proposed that the increase in the damping capacity for the composites (with and without heat treatment) is not due to thermoelasticity, suggesting that other damping mechanisms may be operative for the resulting damping of A356.2/RHA composites.

\subsection{Dislocation damping}

An increase in dislocation density arises in the metallic matrix due to the presence of hard reinforcing particulates. These dislocations are generated due to thermal mismatch strain caused due to the difference in coefficient of thermal expansion values between the metal matrix and hard reinforcing phase. As mentioned earlier the coefficient of thermal expansion (CTE) of the matrix (A356.2) is $21.4 \times 10^{-6} /{ }^{\circ} \mathrm{C}$ and the reinforcement particulates (RHA) is $10.1 \times 10^{-6} /{ }^{\circ} \mathrm{C}$. Thus the dislocation density generated can be quite significant at the interface and can be predicted using the model of Taya and Arsenault (1989) based on prismatic punching of dislocations at a ceramic particulate. The dislocation density $\rho$ at the interface is given by (4)

$$
\rho=\frac{B \varepsilon V_{\mathrm{f}}}{b d\left(1-V_{\mathrm{f}}\right)},
$$

where $B$ is a geometric constant that depends on the aspect ratio (it varies between 12 for equiaxed particulate and 4 for whisker-like particulate), $\varepsilon$ the thermal mismatch strain (the product of temperature change, $\Delta T$, during solidification of MMCs and CTE difference, $\Delta \alpha$, between reinforcement and matrix), $V_{\mathrm{f}}$ the volume fraction of the ceramic reinforcement, $b$ the burgers vector, $d$ the grain diameter of reinforcements. The volume fraction $\left(V_{\mathrm{f}}\right)$ is given by $(5)$

$$
V_{\mathrm{f}}=\frac{\rho_{\mathrm{Al}}-\rho_{\mathrm{mmc}}}{\rho_{\mathrm{Al}}-\rho_{\mathrm{RHA}}},
$$

where $\rho_{\mathrm{Al}}$ is the density of A356.2, $\rho_{\mathrm{mmc}}$ the density of composites and $\rho_{\mathrm{RHA}}$ the density of rice husk ash. Based on (5) and (6) the dislocation density for different experimental samples were calculated with an assumption for the burgers vector of $0.32 \mathrm{~nm}$ for Al (Taya and Arsenault 1989) and are tabulated in table 3 .

Granato-Lucke mechanism is a well-accepted theory that explains the damping mechanism by dislocations. When a dislocation is pinned by two obstacles such as the impurity atoms, ceramic particulates or antiplane dislocations, it
Table 3. Theoretical results of unreinforced and Al/RHA composites.

\begin{tabular}{lccc}
\hline $\begin{array}{l}\text { RHA } \\
\text { weight }(\%)\end{array}$ & $\begin{array}{c}\text { Volume } \\
\text { fraction }\left(V_{\mathrm{f}}\right)\end{array}$ & $\begin{array}{c}\text { Estimated dislocation } \\
\text { density }(\rho)\left(\mathrm{m}^{-2}\right)\end{array}$ & $\begin{array}{c}\mathrm{CTE} \alpha \exp \\
\left(\times 10^{-6} /{ }^{\circ} \mathrm{C}\right)\end{array}$ \\
\hline 0.0 & - & - & $21.4 \times 10^{-6} /{ }^{\circ} \mathrm{C}$ \\
4.0 & 0.028 & $8.81 \times 10^{10}$ & $19.3 \times 10^{-6} /{ }^{\circ} \mathrm{C}$ \\
6.0 & 0.065 & $3.96 \times 10^{11}$ & $17.6 \times 10^{-6} /{ }^{\circ} \mathrm{C}$ \\
8.0 & 0.086 & $7.32 \times 10^{11}$ & $16.3 \times 10^{-6} /{ }^{\circ} \mathrm{C}$ \\
\hline
\end{tabular}

behaves like an elastic vibrating string. Under low amplitude of the applied cyclic load the string vibrates to dissipate energy. Thus at low strain amplitude, damping is independent of strain amplitude and is dependent only on the frequency while at high strain amplitude the damping nature increases tremendously due to breakaway of the dislocations from their pinned positions due to excessive bowing. The dislocation-based damping is mainly due to frequency dependent part and is expressed as follows:

$$
Q^{-1}=\frac{a_{0} B \Lambda L^{4} \omega^{2}}{\pi^{2} C b^{2}},
$$

where $a_{0}$ is a numerical factor of order $1, B$ the damping constant, $\omega$ the operating frequency, $L$ the effective dislocation loop length which depends on the pinning distance, $C$ the dislocation line tension $\left(\approx 0.5 \mathrm{~Gb}^{2}\right), G$ the shear modulus, $b$ the burgers vector and $\Lambda$ the total dislocation density. This shows that the damping depends on the dislocation density, frequency of cyclic stress and burgers vector, $b$, of the bulk material. Thus increased density of dislocation due to presence of hard RHA particulates in the aluminum matrix contributes towards increased dislocation-based damping characteristics in the MMC. It may also be noted that the dislocation density increases with RHA weight percentage and thus an increase in damping of the aluminum matrix was expected due to increase in RHA weight percentage.

\subsection{Generation of plastic zone}

In particulates reinforced metal matrix composites (PRMMCs), inclusion of the hard ceramic particulates causes high residual stresses around the particulates in the form of an annular plastic zone due to large difference in the coefficient of thermal expansion of metallic matrix and ceramic reinforcement, which are responsible for the generation of plastic zone at the particulate/matrix interface. CTE for RHA is $10.1 \times 10^{-6} /{ }^{\circ} \mathrm{C}$ and for A 356.2 alloy is $21.4 \times 10^{-6} /{ }^{\circ} \mathrm{C}$. Based on the works of Dunand and Mortensen (1991) with an assumption of elastic perfectly plastic material behaviour, size of the plastic zone $C_{\mathrm{s}}$ can be estimated as:

$$
C_{\mathrm{s}}=r_{\mathrm{s}}\left[\frac{\Delta \alpha E \Delta T}{(1-v) \sigma_{y}}\right]
$$


Table 4. Theoretical results of unreinforced and Al/RHA composites.

\begin{tabular}{lcc}
\hline $\begin{array}{l}\text { RHA } \\
\text { weight }(\%)\end{array}$ & $\begin{array}{c}\mathrm{CTE} \alpha_{\text {exp }} \\
\left(\times 10^{-6} /{ }^{\circ} \mathrm{C}\right)\end{array}$ & $\begin{array}{c}\text { Radius of plastic } \\
\text { zone }(\mu \mathrm{m})\end{array}$ \\
\hline $0 \cdot 0$ & $21.4 \times 10^{-6} /{ }^{\circ} \mathrm{C}$ & - \\
$4 \cdot 0$ & $19.3 \times 10^{-6} /{ }^{\circ} \mathrm{C}$ & 17.64 \\
$6 \cdot 0$ & $17.6 \times 10^{-6} /{ }^{\circ} \mathrm{C}$ & 31.92 \\
8.0 & $16.3 \times 10^{-6} /{ }^{\circ} \mathrm{C}$ & 42.84 \\
\hline
\end{tabular}

where $\Delta \alpha$ the difference between CTEs of metallic matrix and reinforcement, $\Delta T$ is the temperature difference, $E$ and $v$ are the matrix elastic modulus and Poisson's ratio, $\sigma_{\mathrm{y}}$ the matrix yield stress and $r_{\mathrm{s}}$ the particulate radius. Thus for the present experiment samples containing RHA particulates, the plastic zone radius is calculated with a basic assumption of spherical particle shape using (7) and the corresponding values are listed in table 4.

Recently based on mechanical spectroscopic studies, Carreno-Morelli et al (2000), proposed a simplistic model to determine the damping due to plastic zone as follows:

$$
\tan \delta=\frac{f_{\mathrm{zp}} G_{\mathrm{c}} \int \sigma \mathrm{d} \varepsilon}{\pi \sigma_{0}^{2}},
$$

where $f_{\mathrm{zp}}$ is the plastic zone volume fraction, $G_{\mathrm{c}}$ the shear modulus of the composite sample, $\sigma_{0}$ the alternating shear stress amplitude and $\varepsilon$ the corresponding strain acting on the specimen. Thus it is clear from (8) that the damping depends directly on the volume fraction of plastic zone and strain amplitude. Also it is clear that the overlapping of plastic zones can occur for situations when the plastic zone is larger with smaller inter-particulate distance which is possible as the weight percentage increases.

\subsection{Effect of T6 heat treatment on damping behaviour of A356.2/RHA composites}

In particulate-reinforced metal matrix composites, there are high residual stresses around the particulates because of large difference in the thermal expansion coefficient of metal and reinforcements, which are responsible for the generation of high dislocation density at matrix. For A356-2/RHA composites, $\Delta T$ of T6-treated composites is the smallest, and $\Delta T$ of foundry composites is the largest. The dislocation density of T6-treated composites is the smallest, but its effective length of movable dislocation is the longest. The investigative result of Yamashita (2000) also revealed that the dislocation density of heat-treated composites is lower than that of the foundry composites because the dislocation recombines at solution temperature. The effective length of dislocation in heat treated composites is longer than that of the foundry composites. According to the Granato-Lucke mechanism, the damping capacity of materials is a function of the dislocation density and effective length of movable dislocation. Therefore, the damping capacity of heat-treated composites is larger than that of the foundry composites. Furthermore, heat treatment can decrease interface bonding strength and enlarge the de-bonding of reinforcement and the matrix and this will help in further increase in the damping capacity. Moreover as the de-bonding areas were enlarged after heat treatment because of thermal mismatch, the friction in interface area is enhanced and can dispatch much energy in vibration, which is considered as the reason why damping peaks for heat treated composites at certain frequencies.

\section{Conclusions}

Damping measurements have been studied for composites with and without heat treatment of different weight $\%$ of the reinforcement using dynamic mechanical analyser at frequencies ranging from $1 \mathrm{~Hz}$ to $25 \mathrm{~Hz}$ with a static load of $50 \mathrm{~N}$ and a dynamic load of $40 \mathrm{~N}$ under three-point bending test mode. Composites exhibit higher damping capacity than the unreinforced alloy and increases with the increase in weight percent of the reinforcement. The heat treated composites exhibit higher damping capacity than the untreated composites and increases with the increase in weight $\%$ of the reinforcement and loss in the storage modulus was observed and further decreases with the increase in the weight $\%$ of reinforcement. The damping mechanism ascribed to generation of plastic zone and dislocation damping for the composites.

\section{Acknowledgement}

The authors would like to thank Gitam University, Visakhapatnam, for financial support under a minor research scheme.

\section{References}

Bishop J E and Kinra V K 1995 Metall. Mater. Trans. A-Phys. Metall. Mater. Sci. 262773

Carreno-Morelli E, Urreta S E and Schaller R 2000 Acta Mater. 48 4725

Dunand D C and Mortensen A 1991 Acta Metall. Mater. 39416

Jia Chunlei 2007 Mater. Design 281711

Nelson D J and Hancock J W 1978 J. Mater. Sci. 132429

Rohatgi P K, Nath D, Singh S S and Keshavaram B N 1994 J. Mater. Sci. 295975

Srikanth N and Gupta M 2002 Mater. Res. Bull. 371149

Sudarshan K and Surappa M K 2008 Mater. Sci. Eng. A Struct. Mater. 480117

Taya M and Arsenault R J 1989 Metal matrix compositesthermomechanical behaviour (New York: Pergamon Publishers)

Wang C and Zhu Z 1998 Scr. Mater. 381739

Wu G H, Dou Z Y, Jiang L T and Cao J H 2006 Mater. Lett. 602945

Yamashita S 2000 J. Nucl. Mater. 647283 